\begin{tabular}{|c|l|}
\hline Title & Pressure dependence of structural and electronic properties of polysilane alloys \\
\hline Author(s) & Tanaka, Keiji; Nitta, Shoji \\
\hline Citation & $\begin{array}{l}\text { PHY SICAL REVIEW B, 39(5), 3258-3264 } \\
\text { https://doi.org/L0.1103/PhysRevB.39.3258 }\end{array}$ \\
\hline Issue Date & 1989-02 \\
\hline Doc URL & http://hdl.handle.net/2115/5793 \\
\hline Rights & Copyright $\odot 1989$ A merican Physical Society \\
\hline Type & article \\
\hline File Information & PRB39-5.pdf \\
\hline
\end{tabular}

Instructions for use 


\title{
Pressure dependence of structural and electronic properties of polysilane alloys
}

\author{
Keiji Tanaka \\ Department of Applied Physics, Faculty of Engineering, Hokkaido University, Sapporo, Hokkaido 060, Japan \\ Shoji Nitta \\ Department of Electronics and Computer Engineering, Faculty of Engineering, Gifu University, \\ 1-1 Yanagido, Gifu 501-11, Japan \\ (Received 13 May 1988; revised manuscript received 28 September 1988)
}

\begin{abstract}
Pressure effects on structure and physical properties in quasi-one-dimensional $a$-Si:H films consisting of polysilane molecules and $a$-Si clusters have been investigated up to $100 \mathrm{kbar}$. X-ray diffraction patterns for the film at 1 atm show a prominent first sharp diffraction peak, which shifts to higher angles with decreasing intensity upon pressurizing. The macroscopic volume is compressed dramatically below $50 \mathrm{kbar}$. At higher pressures, the structural changes become less salient, and by contrast, the band-gap energy decreases remarkably with a pressure coefficient of -8 $\mathrm{meV} / \mathrm{kbar}$. These pressure-induced changes are discussed in comparison with characteristics in three-dimensional $a$-Si films and in chalcogenide glasses. Essential roles of low-dimensional structures and $\sigma$-electron states in polysilane molecules are suggested.
\end{abstract}

\section{INTRODUCTION}

Polysilane $\left(\mathrm{SiH}_{2}\right)_{n}$ is an attractive model element for studying condensed matter because it bridges the gap between tetrahedrally coordinated three-dimensional (3D) $\mathrm{Si}$ and chainlike organic polymers such as polyethylene and polyacethylene. In addition, it is of interest to compare the properties with those in chalcogenide glasses, which seem to be composed with entangled chains or distorted layer molecules. ${ }^{1}$ Nonetheless, we have not been able to obtain pure polysilane samples.

Recently, it has become possible to prepare lowdimensional hydrogenated amorphous $\mathrm{Si}(a-\mathrm{Si}: \mathrm{H})$ films. Wolford et $a l .{ }^{2}$ have demonstrated that $a-\mathrm{Si}: \mathrm{H}$ alloys, deposited onto low-temperature substrates by plasma decomposition of disilane $\mathrm{Si}_{2} \mathrm{H}_{6}$ or by the homogeneous chemical-vapor-deposition method of monosilane $\mathrm{SiH}_{4}$, have wider optical-band-gap energies than those in 3D $a$ $\mathrm{Si}: \mathrm{H}$ films prepared by using conventional glow-discharge reactions. On the basis of hydrogen-content measurements and infrared-absorption spectrum analysis, polysilane chain assemblies have been envisaged for the microstructure of the novel binary materials. They have argued further that glow discharge of monosilane under any deposition conditions is not feasible to prepare films containing the one-dimensional (1D) molecules.

Similar $a$-Si:H films have also been deposited using a capacitively coupled glow-discharge reactor by Furukawa and Matsumoto ${ }^{3}$ and an inductively coupled chamber by Nitta et $a l .{ }^{4}$ The novel $a-\mathrm{Si}: \mathrm{H}$ films have the band-gap energy of $1.9-2.5 \mathrm{eV}$ depending on preparation conditions, in contrast with the band-gap energy of $1.1-1.7 \mathrm{eV}$ in conventional 3D films. ${ }^{5}$ Takeda et al. ${ }^{6,7}$ have analyzed the electronic structure using a molecular-orbital method, predicing the band-gap energy of an isolated polysilane chain to be $3.1 \mathrm{eV}$. In order to interpret the difference $(0.6-1.2 \mathrm{eV})$ between the experimental and theoretical band-gap energies, Matsumoto et al. ${ }^{8}$ have adopted a 3D quantum-well model for the structures. They conjecture that the deposited films are microscopically heterogeneous, consisting of 3D $a$-Si clusters embedded in polysilane chain matrices. In such structures, 3D $a$-Si clusters form quantum wells with depths of 1-2 $\mathrm{eV}$, and the optical-absorption edge may be governed by these wells. Taking the energy gaps of the respective regions into account, they deduced that the dimension of the Si clusters was of the order of $10 \AA$. In light of this structural model, the novel $a-\mathrm{Si}: \mathrm{H}$ films may be referred to as "polysilane alloys" or "quasi-1D $a$-Si:H.",3,4

It has been shown that pressure studies give fruitful insights into the bonding topology in amorphous semiconductors. ${ }^{1,5,9,10}$ A principal role of hydrostatic compression is to decrease the interatomic distance. In lowdimensional materials, there exist two kinds of atomic bonds, inter- and intramolecular bonds, characterized, respectively, by van der Waals and covalent forces. The van der Waals bonds are weaker by 1-2 orders of magnitude than the covalent bond, and therefore hydrostatic pressure preferentially compresses the intermolecular distance. The macroscopic volume of materials is, as a consequence, greatly reduced. When materials are subjected to high pressures, changes in covalent-bond geometries and structural transformations such as pressure-induced polymerization may occur. On the other hand, in 3D rigid networks pressure effects are much smaller. Hence, pressure studies for polysilane alloys consisting of $1 \mathrm{D}$ and $3 \mathrm{D}$ clusters seem attractive to reveal the nature, whereas no works have been reported, except a preliminary result by the present authors. ${ }^{11}$

In the present paper, we examine changes in $x$-ray diffraction patterns, the density, and the opticalabsorption edge of quasi-1D $a$-Si:H films subjected to hydrostatic pressure. In Sec. II experimental details are described. Section III provides results and discussion for 
properties of the films under pressure, in comparison with those for chalcogenide glasses, $a-\mathrm{Si}$, and 3D $a-\mathrm{Si}: \mathrm{H}$ films. Finally, we summarize arguments in Sec. IV.

\section{EXPERIMENTS}

Polysilane alloy films were deposited onto glass substrates of Corning 7059 by glow discharge of disilane gas in a $13.56-\mathrm{MHz}$ inductively coupled system. The rf power, total gas pressure, and substrate temperature were, respectively, $15 \mathrm{~W}, 1$ Torr, and room temperature. The film thicknesses were approximately 1 and $10 \mu \mathrm{m}$. The texture of the films was rough and the surface was not optically flat. Free-standing films for hydrostatic pressure experiments were obtained by peeling off from the substrates. The hydrogen content of the films was not evaluated precisely. However, the previous study ${ }^{4}$ using infrared-absorption spectroscopy and hydrogen-evolution measurements for films deposited under similar conditions has confirmed that the hydrogen content is approximately 50 at. \%, substantial parts of which are incorporated in a form of polysilane chains. Oxidation of films was conceivable: ${ }^{4}$ nonetheless, the effects were imperceptible for the present results. ${ }^{12}$

The free films were pressurized at room temperature in a gasketed diamond-anvil cell along with the methanolethanol mixture. ${ }^{13}$ The gaskets having circular holes 0.3 $\mathrm{mm}$ in diameter were made of $0.15-\mathrm{mm}$-thick Inconel plate. The generated pressure was calibrated with the wavelength shift of the ruby $R_{1}$ peak. ${ }^{13}$ X-ray diffraction patterns, optical transmittances, and linear dimensions of the film chips under pressure were measured in situ.

Figure 1 shows a drawing of the experimental setup for

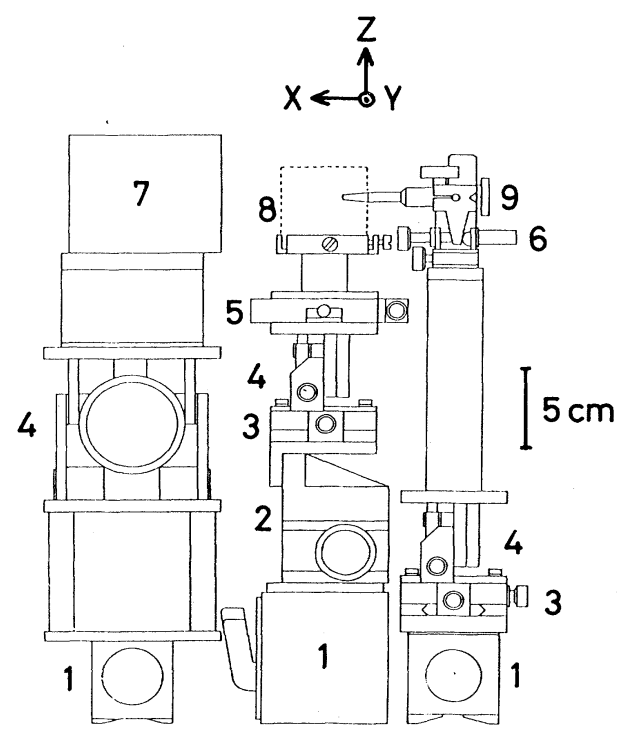

FIG. 1. A side view of the x-ray diffraction system. 1, magnetic stand; position adjuster along the $x$ direction; 3 , position adjuster along $y$ direction; 4 , position adjuster along $z$ direction; 5 , rotating adjuster in $x-y$ plane; 6 , tilt adjuster around $y$ and $z$ axes; 7, PSPC; 8, diamond-anvil cell; 9, collimator. An x-ray target and a monochromator are located at the right-hand side out of this figure. $\mathrm{x}$-ray diffraction experiments. The arrangement is essentially the same with that developed by Fujii et al., ${ }^{14}$ whereas details are largely different. A high-luminous rotating Mo source $\left[(60 \mathrm{kV}) \times(40 \mathrm{~mA}) /\left(0.01 \mathrm{~mm}^{2}\right)\right]$ and a graphite monochromator, located out of the right-hand side of the figure, were employed for obtaining a beam of $\lambda=0.711 \AA$. The beam passes through a collimator $5 \mathrm{~cm}$ long having pinholes $0.1 \mathrm{~mm}$ in diameter at both ends, and irradiated the sample in the diamond-anvil cell. Positions of the collimator and the cell were critically adjusted using several manipulating devices shown in Fig. 1. Transmitted and diffracted beams from the sample were detected by a linear position-sensitive proportional counter (PSPC) filled with a flowing gas mixture of krypton and methane. The electrical signal from the counter was processed by a position analyzer, a pulse-height analyzer, and a personal computer. Exposure time was $5000 \mathrm{~s}$ at each pressure, and the maximum intensity diffracted from the sample was about 500 counts. The data were background subtracted and smoothed for improving statistical accuracy.

Some comments may be needed for details of the diffraction experiment. The first regards the sample thickness. Taking the absorption coefficient of Si for Mo $K \alpha$ line into account, an appropriate sample thickness is estimated to be about $1 \mathrm{~mm}$. However, samples in pressure experiments using diamond-anvil cells should be thinner than the gaskets, and in the present investigation piles of $10-\mu \mathrm{m}$-thick films, effectively $50-100 \mu \mathrm{m}$, were employed. Second, the horizontal scale of the diffraction patterns (Fig. 2) corresponds to the linear position of the detector and, hence, the scale is regular neither in angle $\theta$ nor in scattered wave number $Q(=4 \pi \sin \theta / \lambda)$. The scale is calibrated using diffraction peaks from Al foil.

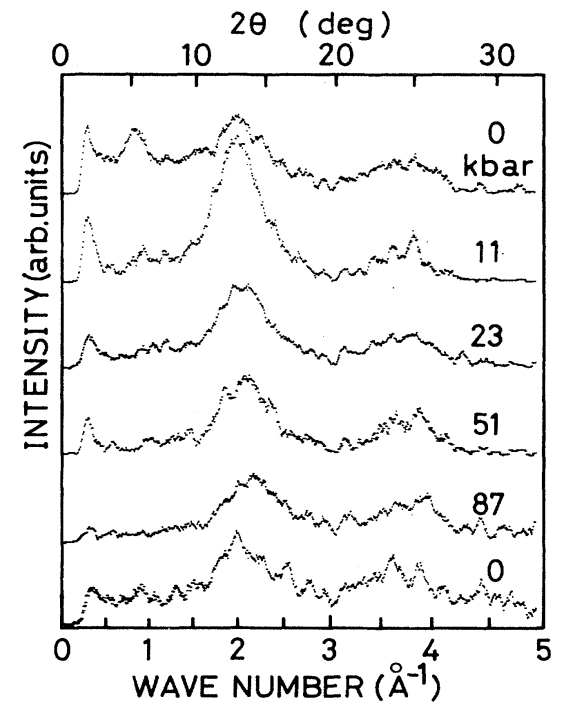

FIG. 2. X-ray diffraction patterns of $a$-Si:H under hydrostatic pressure. The pressure is increased from the upper plot to the lower one, and released to $1 \mathrm{~atm}$ in the bottom profile, which is noisy because the sample is effectively thinner. X-ray intensity below $0.3 \AA^{-1}$ is filtered. 
The third point is concerned with the angular resolution. The distance between the sample and the detector was adjusted at about $10 \mathrm{~cm}$, and the counter was arranged so that the direct beam impinged nearly normally on it. In addition, the detector window has a height of $1 \mathrm{~cm}$. Accordingly, because of oblique incidence and the finite window width, the peaks diffracted at high and low angles were distorted and broadened. A simple geometrical analysis showed that the distortion and broadening affected by these factors were less than $\pm 1.5^{\circ}$. This magnitude may be neglected on evaluating positions of peaks diffracted from amorphous materials, since the halo peaks are substantially wider.

The linear dimension of a sample chip contained in the pressurized cell was measured directly using an optical microscope attached with a $10 \times$ objective lens and a microscale. $^{15}$ The accuracy of the measurement was \pm 1 $\mu \mathrm{m}$, approximately $\pm 1 \%$, since a typical lateral dimension $L$ of samples was $100 \mu \mathrm{m}$. The in-plane linear compressibility $\Delta L / L$ was evaluated from the results. If we tentatively assume an isotropic contraction, which may not be retained for glow-discharge films, then $3(\Delta L / L)=\Delta V / V=-\Delta \rho / \rho$, where $V$ is the volume and $\rho$ is the density and, hence, the bulk modulus or the bulk compressibility can be estimated. The density of the films at $1 \mathrm{~atm}$ was determined by measuring the film thickness and the $\mathrm{x}$-ray-absorption coefficient for Co $\mathrm{K} \alpha$ line.

The optical transmittance of thin samples $1 \mu \mathrm{m}$ thick was inspected using a microscope spectral system, ${ }^{16}$ which was also used to monitor the ruby $R_{1}$ photoluminescence. Films of about $10 \mu \mathrm{m}$ thickness could not be evaluated optically because of substantial light scattering and, accordingly, examination of the Urbach tail were impossible. (The scattering appeared mostly to be a bulk effect, so that for the thinner films transmittance could be measured.)

\section{RESULTS AND DISCUSSION}

\section{A. Structural}

The density of films was $1.5 \pm 0.2 \mathrm{~g} / \mathrm{cm}^{3}$, which was used to estimate the hydrogen concentration. Fritzsche et al. ${ }^{17}$ have suggested a linear correlation between the density and the hydrogen content for glow-discharged $a$ $\mathrm{Si}: \mathrm{H}$, and Mosseri et al. ${ }^{18}$ have demonstrated that the proportionality can also be applied to $a-\mathrm{Si}: \mathrm{H}$ films produced by different preparation procedures. We may, therefore, follow the Fritzsche correlation, which gives for the present samples the hydrogen content of about 50 at. \%, comparable to the previous result. ${ }^{4}$

Figure 2 shows the pressure dependence of $x$-raydiffraction patterns for quasi-1D $a-\mathrm{Si}: \mathrm{H}$ films. At $1 \mathrm{~atm}$, in the investigated $Q$ region up to $6 \AA^{-1}$, we see three peaks at $Q=0.80,2.0$, and $3.8 \AA^{-1}$. A weak peak at 5.5 $\AA^{-1}$, not shown in the figure, was also detected. The three peaks at $2.0,3.8$, and $5.5 \AA^{-1}$ appear similarly in diffraction patterns for $a$-Si and 3D $a$-Si:H films, ${ }^{5,18}$ but such a strong peak at around $1 \AA^{-1}$ has never been detected previously for $a-\mathrm{Si}: \mathrm{H}$ films.

Figure 3 summarizes some quantitative features of



FIG. 3. Pressure dependencies of characteristic features of the first (solid lines) and second peak (dashed line) in $x$-ray diffraction patterns. $t, I$, and $Q$ denote, respectively, the correlation length evaluated from the half-width through the Scherrer equation, the intensity normalized with the peak intensity at $3.8 \AA^{-1}$, and the scattered wave number.

pressure-induced effects in the $x$-ray profiles. Angular and intensity changes in the $0.8-\AA^{-1}$ peak are dramatic, most notably being its intensity decrease and disappearance with the peak shift to higher $Q$ values. The shift of the $2-\AA^{-1}$ peak is substantially smaller, whereas the peak intensity changes anomalously. The intensity normalized with respect to that of the $3.8-\AA^{-1}$ halo reaches a maximum at a pressure less than $10 \mathrm{kbar}$, and then weakens gradually. ${ }^{19}$ The correlation length $t$, evaluated for the $2-\AA^{-1}$ peak using the Scherrer equation

$$
t=\lambda /(B \cos \theta),
$$

where $B$ is the half-height width, is nearly constant up to 30-40 kbar and then decreases. Pressure-induced changes in the 3.8 - and $5.5-\AA^{-1}$ peaks were not appreciable.

The present $\mathrm{x}$-ray profiles limited to the low- $Q$ region make precise radial-distribution-function (RDF) analyses hardly possible and, instead, we discuss origins of the peaks on the basis of previous knowledge and the Debye scattering equation:

$$
I(Q)=\sum_{m, n} f_{m} f_{n} \sin \left(Q r_{m n}\right) /\left(Q r_{m n}\right),
$$

where $f$ is the atomic scattering factor and $r$ is the separation of a pair of atoms identified by the subscripts $m$ and $n$. Since the atomic scattering factor of $\mathrm{H}$ atoms is less than $10 \%$ of that for $\mathrm{Si}$ atoms, the scattering intensity from $\mathrm{H}$ atoms may be neglected. The scattering factor of $\mathrm{Si}$ undergoes only a smooth modification with the scattering angle and, accordingly, Eq. (2) can be used to 
predict that atomic pairs separated at $r$ give the first and weaker second peaks approximately at $Q_{1}$ and $Q_{2}$, expressed as

$$
Q_{1} r=7.72 \text { and } Q_{2} r=14.1 \text {. }
$$

The peak at $2 \AA^{-1}$ indicates existence of atomic pairs having a correlation distance of $3.9 \AA$. It seems reasonable to connect this peak with the second-nearestneighbor distance $3.8-4.2 \AA$ between $\mathrm{Si}$ atoms, which is estimated through the theoretical analysis for a single polysilane chain. ${ }^{6}$ The pressure-induced changes of this peak reflect bond-angular fluctuation, because covalentbond lengths are little modified by moderate compression.

Equation (3) implies that the peak at $0.80 \AA^{-1}$ is related to pairs of $\mathrm{Si}$ atoms being located approximately $9.7 \AA$ apart. This low- $Q$ peak can be ascribed to interchain correlation between polysilane chain molecules for the following two reasons.

The first concerns its position. We note that in amorphous or melted polyethylene $\left(\mathrm{CH}_{2}\right)_{n}$ the interchain correlation produces a peak at $Q=1.3 \AA^{-1} \cdot{ }^{20,21}$ which corresponds to the interchain distance of $5.9 \AA$. It is known that crystalline polyethylene has $\mathrm{C}-\mathrm{C}$ skeleton chains forming planar zigzag structures, ${ }^{22}$ and it is suggested theoretically that polysilane chains have similar planar skeletons. ${ }^{6,7}$ Using the Pauling atomic radii for $\mathrm{Si}$, $\mathrm{C}$, and $\mathrm{H}$ atoms, we can estimate the mean distances between chain axes to be $5.4 \AA$ for polyethylene and $6.8 \AA$ for polysilane. The separations are necessarily expanded in amorphous materials containing free volume and, therefore, these values appear comparable to the experimental values of 5.9 and $9.7 \AA$.

Secondly, the pressure dependence of the low- $Q$ peak in comparison with characteristics in other materials strongly suggests its intermolecular nature. As for the peak shift, we compare the result with the data obtained for crystalline polyethylene, since as far as the authors' knowledge extends no pressure $x$-ray studies for noncrystalline polyethylene have been reported. The result reported by Yamamoto et al. ${ }^{23}$ indicates that the average distance between polyethylene chains shrinks with a rate of $0.5 \% / \mathrm{kbar}$. In contrast, the present dependence shown in Fig. 3 gives the rate of $(0.6 \pm 0.2) \% / \mathrm{kbar}$, nearly the same as the organic polymer value. The change responsible for covalent bonds is, however, smaller by at least 1 order of magnitude than these coefficients, $5,10,23,24$ and accordingly the origin can be ascribed to weak intermolecular forces.

As for the pressure dependence of the peak intensity, we note that it resembles the behavior of the first sharp diffraction peak (FSDP) in chalcogenide glasses. ${ }^{10}$ In both materials, the peaks weaken dramatically with increasing pressure. This characteristic is interpreted as resulting from enhanced distortion of molecular substances. ${ }^{10}$ As shown in Figs. 2 and 3, the reduced intensity is not recovered when the sample is released from 87 kbar to $1 \mathrm{~atm}$. The recovery was incomplete, even when the sample was depressurized from $10 \mathrm{kbar}$. Such a hysteresis effect is also observed in the FSDP in chalcogenide glasses. ${ }^{10}$ We may, therefore, regard the low- $Q$ peak in
$a-\mathrm{Si}: \mathrm{H}$ films as a FSDP, in the context that the nature is intermolecular, similarly in chalcogenide glasses. ${ }^{25}$

It has been argued that the peak at $0.8 \AA^{-1}$ arises from the intermolecular correlation between polysilane chains, while details of the interchain correlation are not known. To consider the structure, it may be fruitful to follow extensive studies made for noncrystalline polyethylene. ${ }^{21}$ However, only on the basis of diffraction experiments, it is difficult to predict, for instance, whether the chains form random-coil structures or some kinds of bundled configurations. Hence, in the following we try to delineate a picture of $a-\mathrm{Si}: \mathrm{H}$ structure, taking several insights into account.

The volume fractions of the polysilane and $a$-Si clusters can be estimated as follows. The macroscopic density $\rho$ of the film may be expressed as

$$
\rho=(1-x) \rho_{c}+x \rho_{p},
$$

where $\rho_{c}$ and $\rho_{p}$ are the densities of $a-\mathrm{Si}$ and polysilane regions and $x$ is the volume fraction of polysilane clusters. Voids are tentatively neglected here. We may approximate $\rho_{c}$ with that of crystalline $\mathrm{Si}, 2.3 \mathrm{~g} / \mathrm{cm}^{3}{ }^{26}$ The interchain separation $9.7 \AA$ between polysilane chains together with the predicted chain configuration ${ }^{6}$ implies $\rho_{p} \simeq 0.3 \mathrm{~g} / \mathrm{cm}^{3}{ }^{27}$ Then, the experimental density $\rho=1.5$ $\mathrm{g} / \mathrm{cm}^{3}$ reads $x \simeq 0.4$.

The half-height width $B$ of the FSDP at 1 atm shown in Fig. 2 suggests, through the Scherrer equation [Eq. (1)] applicable also to chain clusters, ${ }^{28}$ that the dimension $t_{p}$ of correlated polysilane regions is approximately $15 \AA$. (Note that this value is the lower limit, since as was mentioned in Sec. II the diffraction patterns are broadened by the spatial resolution of the $x$-ray detector.) In contrast, the degree of polymerization $n$ for the polysilane molecule $\left(\mathrm{SiH}_{2}\right)_{n}$ in glow-discharged $a$-Si:H films is predicted to be $n \lesssim 10$ by Furukawa and Matsumoto ${ }^{3}$ analyzing infrared spectra. Using the covalent-bond length $2.4 \AA$ and angle $110^{\circ}$ of $\mathrm{Si}$ skeletons, ${ }^{6}$ we can read $n \lesssim 10$ as the chain length $l \lesssim 20 \AA$. Therefore, a random-coil model which requires $l \gg t_{p}$ cannot be adopted for the present materials. Alternatively, we may envisage nearly straight chains lying in correlated regions, with an average interchain separation of $\sim 10 \AA$. Whether the chain fragments are aligned or randomly oriented, however, cannot be inferred. ${ }^{21}$

On the basis of the two-phase structural model ${ }^{8}$ and the above speculative argument, we may visualize the structure at 1 atm as shown in Fig. 4. The film is com-

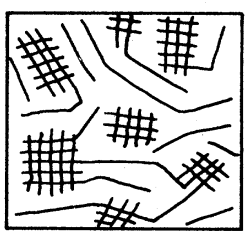

1 atm

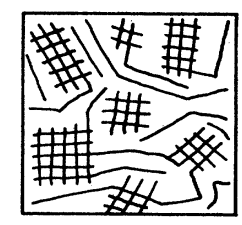

$50 \mathrm{kbar}$

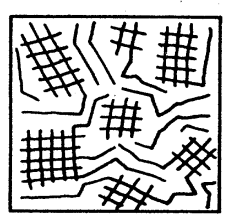

$100 \mathrm{kbar}$
FIG. 4. Microscopic structural models of $a-\mathrm{Si}: \mathrm{H}$ films as a function of pressure. Polysilane and $a$-Si clusters are represented by solid lines and square lattices. 
posed of $a$-Si clusters embedded in polysilane regions having the medium-range correlation. The size of $a-S i$ clusters is assumed to be distributed in the order of 10 $\AA$ A. ${ }^{8}$ Voids, probably included, ${ }^{4}$ are not shown in the figure, since there is no information on the void size. Of course, a void as small as $10 \AA$ cannot be distinguished from the free volume between polysilane chains.

It appears that the pressure-induced structural transformation is not monotonic, but has a threshold at around 50 kbar. As shown in Fig. 3, under the threshold, the change in the $2-\AA^{-1}$ peak width is not remarkable. ${ }^{29}$ The FSDP disappears at approximately this value, which indicates that polysilane molecules can no longer be distinguished from $a$-Si clusters with respect to the electron density on a scale of $\sim 10 \AA$. The interchain separation at $50 \mathrm{kbar}$ suggested from the peak position through Eq. (3) is about $7 \AA$.

Hence, we can sketch the pressure-induced structural transformation in polysilane alloys as shown in Fig. 4. Up to $50 \mathrm{kbar}$, the main effects of hydrostatic compression are to crush the voids and to reduce the interchain distance between polysilane molecules from $\sim 10$ to $\sim 7$ $\AA$. The polysilane chains are closely packed, being distorted substantially, but the angular correlation inferred from the second peak is still preserved. Above the critical pressure, the electron density in the medium-range scale becomes spatially homogeneous, and the bond-angle fluctuation in polysilane traces is enhanced with increasing pressure. Disruption and cross-linking of chains may be induced.

Figure 5 shows the macroscopic compression behavior of quasi-1D $a-\mathrm{Si}: \mathrm{H}$, together with those for crystalline $\mathrm{Si}$ (Ref. 26) and 3D $a$-Si:H. ${ }^{10,30}$ We see for the $1 \mathrm{D}$ material a dramatic contraction up to $\sim 50$ kbar and a small change above this pressure. It might be important to mention that the films sometimes cracked when compressed above $50 \mathrm{kbar}$. The samples released from higher pressures were smaller in length by $\sim 1.5 \%$, or densified by $\sim 5 \%$.

The macroscopic characteristics are consistent with the speculation for the microscopic structural changes. The linear compressibility of $0.1 \% / \mathrm{kbar}$ below $50 \mathrm{kbar}$,

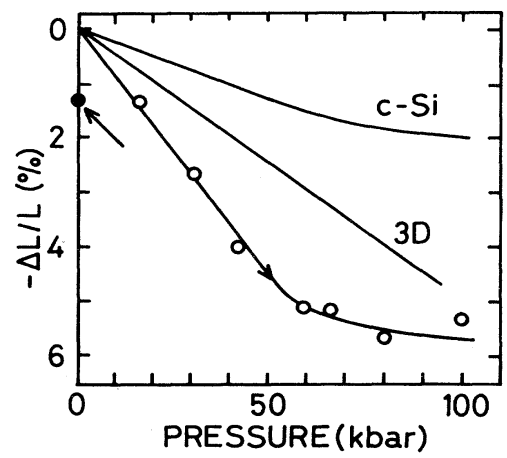

FIG. 5. Fractional changes of linear dimensions in crystalline $\mathrm{Si}, 3 \mathrm{D} a-\mathrm{Si}: \mathrm{H}$, and quasi-1D $a-\mathrm{Si}: \mathrm{H}$ under pressure. A solid circle indicates a densified state of a quasi-1D film released from 100 kbar. or the bulk modulus of $300 \mathrm{kbar}$, is comparable to that of molecular materials. ${ }^{30}$. The deformation arises from shrinkage of voids and the interchain separation between polysilane molecules. In contrast, the bulk modulus above 50 kbar may be rather greater than that of 600 kbar in 3D $a-\mathrm{Si}: \mathrm{H},{ }^{30}$ and similar to that in crystalline $\mathrm{Si}^{26}$ The rigidity can be attributed to networked structures.

\section{B. Electronic}

Figure 6 shows the optical-transmission spectra of quasi-1D $a$-Si:H under pressure. At 1 atm the opticalabsorption edge is located at around $2 \mathrm{eV}$, the value consistent with the previous results. ${ }^{2-4}$ On increasing pressure, the aborption edge shifts to lower energies. Since the precise thickness and the refractive index of the films could not be determined because of rough texture, we were not able to discuss the Tauc energy gap. Instead, we will assume that the photon energy at the $5 \%$ transmittance gives a measure of the optical-band-gap energy. The transmittance value corresponds approximately to $2 \times 10^{4} \mathrm{~cm}^{-1}$ in the absorption coefficient.

Figure 7 shows the the optical-band-gap energy of quasi-1D $a$-Si:H films as a function of pressure. We see that the pressure coefficient of the band-gap energy is always negative; nonetheless the dependence behaves in a quantitatively different way in the high- and low-pressure regions. When pressure is lower than $50 \mathrm{kbar}$, the pressure coefficient is approximately $-1 \mathrm{meV} / \mathrm{kbar}$. Above this pressure, the coefficient becomes smaller, -8 $\mathrm{meV} / \mathrm{kbar}$. When the sample is released from pressure higher than $50 \mathrm{kbar}$, a remarkable hysteresis is observed, as shown in the figure.

In conventional 3D $a$-Si films, the pressure coefficient of negative values ${ }^{5,10,31}$ seems to be governed by two factors. One is the increasing energy splitting between bonding (valence) and antibonding (conduction) bands with decreasing covalent-bond length. In contrast, the absorption edge may be red shifted from pressureenhanced structural randomness, such as fluctuations in the covalent-bond angle. ${ }^{32}$

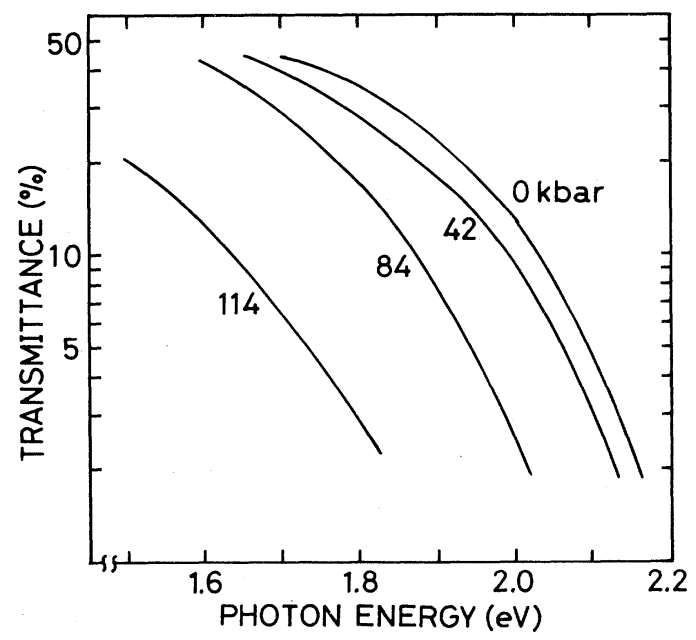

FIG. 6. The optical transmittance of $a$-Si:H under pressure. 


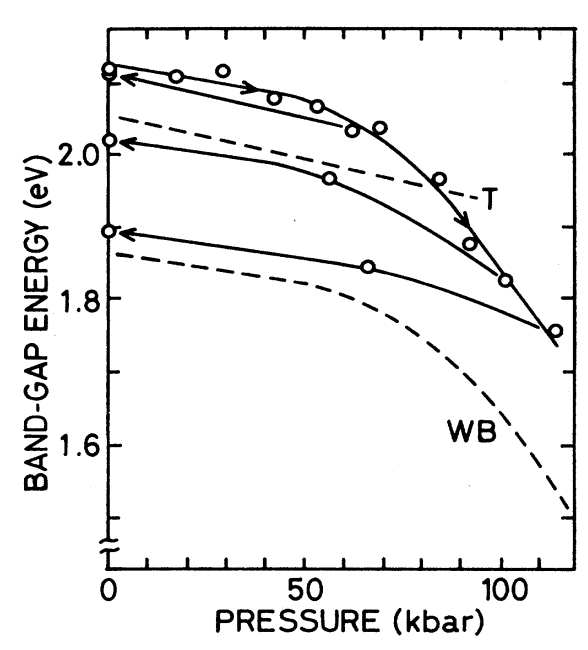

FIG. 7. Changes in the optical-band-gap energy for quasi-1D (solid lines) and 3D (dashed lines) $a-\mathrm{Si}: \mathrm{H}$ films, which are denoted by $T$ (Ref. 10) and WB (Ref. 31).

In order to consider the pressure behavior of the band-gap energy in polysilane alloys, the structural heterogeneity shown in Fig. 4 should be taken into account. Namely, $a-\mathrm{Si}$ quantum wells and polysilane chains, for which Takeda et al. ${ }^{6}$ have theoretically predicted the band-gap energy of $3 \mathrm{eV}$, settled down between the valence band originating from $p$-electron states and the conduction band of $s$-like electrons. . Further, they have evaluated effects of structural modifications, i.e., stretching, bending, branching, and changes in surrounding, on the electronic structure. ${ }^{6,7}$ In the present context, specifically interesting is that the bond bending of polysilane skeletons substantially influences the top of the valence band, and that the band-gap energy decreases with increasing bond angle. Their analysis reveals further the band-gap energy depending sensitively also on changes in $\mathbf{H}-\mathrm{Si}-\mathrm{H}$ angles, while no information on the angular change is available at present. We emphasize, however, that the theoretical analyses do not include the interchain interaction between polysilane molecules. The effect may be small, since there are no $\pi$ electrons, whereas a quantitative evaluation is vitally important to obtain a convincing picture of the electronic changes.

Under the above idea and the pressure-structural variation shown in Fig. 4, we can interpret the pressure dependence of the band-gap energy in quasi-1D $a-\mathrm{Si}: \mathrm{H}$ films as follows. Below $50 \mathrm{kbar}$, the main structural change, namely the shrinkages of free volume between polysilane chains and voids, seems to have little effect on the bandgap energy, because the energy is mostly governed by $a-\mathrm{Si}$ quantum wells. The structural change may decrease the energy gap of polysilane regions, while the effect on the observed energy gap is indirect. The pressure coefficient may be determined from reductions in the quantum-well depths resulting from the energy decrease in polysilane regions and, in addition, from small distortions of $a-\mathrm{Si}$ clusters.
At around $50 \mathrm{kbar}$, the polysilane chains are compressed ultimately, and the changes in the bond angle and the interchain interaction directly govern the decrease in the band-gap energy. Following the theoretical analyses, ${ }^{7,8}$ the increase in the bond-angle fluctuation of $\pm 10 \%$, which may be implied from the change in the half-width of the $2-\AA^{-1}$ diffraction peak, seems to reduce the band-gap energy by $\sim 1 \mathrm{eV}$, comparable to the experimental decrease. The band gap may also be influenced from a creation of dangling bonds. After Pauling, the bond energies of $\mathrm{Si}-\mathrm{H}$ and $\mathrm{Si}-\mathrm{Si}$ are, respectively, 3.0 and $1.8 \mathrm{eV}$, and therefore, $\mathrm{Si}-\mathrm{Si}$ bonds may preferentially be disrupted by local strain.

We should mention, however, that the characteristic band-gap-energy change is not unique to quasi-1D films. Welber and Brodsky ${ }^{31}$ have reported pressure dependencies of the optical-band-gap energy for several $a-\mathrm{Si}: \mathbf{H}$ films, which are supposed to have 3D structures, because the samples were glow discharged from monosilane onto substrates of $250^{\circ} \mathrm{C}$. $^{2}$ Surprisingly, the dependence, also shown in Fig. 7, resembles that for polysilane alloys. By contrast, the results reported by Minomura ${ }^{5}$ and by Tana$\mathrm{ka}^{10}$ for sputtered and glow-discharged 3D a-Si:H show the pressure dependence of -1 to $-3 \mathrm{meV} / \mathrm{kbar}$ in pressure regions lower than $100 \mathrm{kbar}$.

These comparisons indicate, therefore, that the existence of polysilane molecules is not essential to the substantial red shift of the optical-absorption edge. It is known that 3D $a-\mathrm{Si}: \mathrm{H}$ films contain $\mathrm{SiH}$ and $\mathrm{SiH}_{2}$ species, which may segregate around small voids. These hydrogenated species seem to play a similar role to that of polysilane when the samples are compressed. The variety of the pressure dependence in 3D materials may be attributed to different spatial distributions of hydrogenated units and voids.

Finally, a comparative discussion of the pressure effects on the band gaps in $\mathrm{Si}$ alloys and in chalcogenide glasses as well as organic polymers may be of interest. Although chalcogenide glasses show various characteristics of the pressure-induced red shifts, the extremal coefficients amount to $-20 \mathrm{meV} / \mathrm{kbar}$ for most samples. ${ }^{10}$ Low-dimensional organic polymers having $\pi$ electrons such as polyacetylene exhibit the pressure dependence of -12 to $-20 \mathrm{meV} / \mathrm{kbar}^{33}$ By contrast, the value for $a-\mathrm{Si}: \mathrm{H}$, whether the structure may be $1 \mathrm{D}$ or $3 \mathrm{D}$, is at most $-8 \mathrm{meV} / \mathrm{kbar}$, as is shown in Fig. 7. This difference can be attributed to the distinct natures of the conduction and valence bands in the two categories of materials. The band gap of chalcogenide glasses and organic semiconductors is dramatically influenced from the intermolecular interaction through overlapping of $\pi$ electrons forming the valence bands. In $a-\mathrm{Si}: \mathrm{H}$ and $a-\mathrm{Si}$, however, only $\sigma$ bonding electrons exist and, as a consequence, the intermolecular interaction seems relatively smaller. The band gap in these materials is, instead, sensitive to the angular changes in covalent bonds, which need greater strain energy.

\section{SUMMARY}

Pressure effects on structural and electronic properties of quasi-1D $a$-Si:H-alloy films have been studied. The 
one-dimensional nature of polysilane is disclosed in the existence and the pressure dependence of the first sharp diffraction peak, and in the large macroscopic compressibility. These features are comparable to those in lowdimensional chalcogenide glasses.

Electronically, $a-\mathrm{Si}: \mathrm{H}$ films exhibit an appreciable, but still smaller, pressure-induced red shift of the opticalabsorption edge than that in chalcogenide glasses. This electronic change is partly due to the band structure composed of $\sigma$ electrons, seemingly having negligible pressure-enhanced interaction. In this respect, $\mathrm{Si}$ chains are distinct from chalcogenide glasses and organic molecules having $\pi$ electrons, which contribute to drastic reductions in the band-gap energy through intermolecular interaction.
The present study is rudimentary, and extensive investigations are needed to elucidate the properties of lowdimensional $\mathrm{Si}: \mathrm{H}$ films. For instance, detailed diffraction studies both in wide- and small-angle regions, are valuable to obtain convincing structural pictures.

\section{ACKNOWLEDGMENTS}

The authors would like to thank Professor K. Matsushige for valuable suggestions on organic polymers and Dr. N. Takahashi for the use of the graphite monochromator. The $\mathrm{x}$-ray experiment was carried out at the High Brilliance X-Ray Laboratory at Hokkaido University. The present work was partially supported by the Asahi Glass Foundation for Industrial Technology.
${ }^{1}$ R. Zallen, The Physics of Amorphous Solids (Wiley, New York, 1983), Chaps. 3 and 6.

${ }^{2}$ D. J. Wolford, J. A. Reimer, and B. A. Scott, Appl. Phys. Lett. 42, 369 (1983).

${ }^{3}$ S. Furukawa and N. Matsumoto, Solid State Commun. 48, 539 (1983); Phys. Rev. B 31, 2114 (1985).

${ }^{4}$ S. Nitta, M. Kawai, M. Sakaido, I. Murase, and A. Hatano, in Tetrahedrally-Bonded Amorphous Semiconductors, edited by D. Adler and H. Fritzsche (Plenum, New York, 1985), p. 501.

${ }^{5} \mathrm{~S}$. Minormura, in Semiconductors and Semimetals, edited by $\mathrm{J}$. I. Pankove (Academic, Orlando, 1984), p. 273.

${ }^{6}$ K. Takeda, N. Matsumoto, and M. Fukuchi, Phys. Rev. B 30, 5871 (1984).

${ }^{7}$ K. Takeda and N. Matsumoto, J. Phys. C 18, 6121 (1985).

${ }^{8}$ N. Matsumoto, S. Furukawa, and K. Takada, Solid State Commun. 53, 881 (1985).

${ }^{9}$ G. Parthasarathy and E. S. R. Gopal, Bull. Mater. Sci. 7, 271 (1985)

${ }^{10}$ K. Tanaka, J. Non-Cryst. Solids 90, 363 (1987); 97\&98, 391 (1987).

${ }^{11}$ K. Tanaka and S. Nitta, Solid State Commun. 66, 827 (1988).

${ }^{12}$ Optical and compressibility results obtained for fresh samples and for films stored for one month in air were similar.

${ }^{13}$ A. Jayaraman, Rev. Mod. Phys. 55, 65 (1983).

${ }^{14}$ Y. Fujii, O. Shimimura, K. Takemura, S. Hoshino, and S. Minomura, J. Appl. Crystallogr. 13, 284 (1980).

${ }^{15}$ K. Tanaka and J. Maeda, Rev. Sci. Instrum. 57, 500 (1986).

${ }^{16}$ K. Tanaka, Jpn. J. Appl. Phys. 25, 779 (1986).

${ }^{17}$ H. Fritzsche, M. Tanielian, C. C. Tsai, and P. J. Gaczi, J. Appl. Phys. 50, 3366 (1979).

${ }^{18}$ R. Mosseri, J. C. Malaurent, C. Sella, and J. Dixmier, J. NonCryst. Solids 35\&36, 507 (1980).

${ }^{19}$ Note that this maximal ratio of $\sim 5$ is much more intense than that $(\sim 1)$ observed in conventional 3D $a-\mathrm{Si}: \mathrm{H}$ films containing $\mathbf{H}$ atoms of about 10 at. \% (Ref. 5). It is reported that the intensity becomes more prominent with the hydrogen content (Ref. 5), whereas its origin has not been known.

${ }^{20}$ O. Yoda, I. Kuriyama, and A. Odajima, J. Appl. Phys. 49, $5468(1978)$
${ }^{21}$ R. Lovell, G. R. Mitchell, and A. H. Windle, Faraday Spec. Discuss. Chem. Soc. 69, 46 (1979).

${ }^{22}$ D. C. Bassett, Principles of Polymer Morphology (Cambridge University Press, London, 1981), Chap. 1.

${ }^{23}$ T. Yamamoto, H. Miyaji, and K. Asai, Jpn. J. Appl. Phys. 16, 1891 (1977).

${ }^{24}$ R. Keller, W. B. Holzapfel, and H. Schulz, Phys. Rev. B 16, 4404 (1977).

${ }^{25}$ L. Cervinka, J. Non-Cryst. Solids 90, 371 (1987).

${ }^{26}$ American Institute of Physics Handbook, edited by D. E. Gray (McGraw-Hill, New York, 1972).

${ }^{27}$ This value may be too small, since the density of noncrystalline polyethylene is about $0.8 \mathrm{~g} / \mathrm{cm}^{3}$ (Refs. 20 and 21). Under assumptions that polysilane chains form microcrystals, and that two interchain distances along $a$ and $b$ axes correspond to the diffraction peaks at 0.8 and $1.6 \AA^{-1}$, the density is evaluated as $0.8 \mathrm{~g} / \mathrm{cm}^{3}$.

${ }^{28}$ A. M. Hindeleh and D. J. Johnson, Polymer 21, 929 (1980).

${ }^{29}$ It should be mentioned that the behaviors of the $2-\AA^{-1}$ peak are convoluted over $a$-Si and polysilane clusters. The distortion of the peak reflecting angular correlation seems to be governed by structural changes in the polysilane fragments, since 1D materials appear more fragile than 3D clusters. A comparison of the present results with that for $a$-Si reinforces this conjecture. The $2-\AA^{-1}$ peak in $a$-Si films also shows a slight shift when subjected to hydrostatic pressure (Ref. 5). Nonetheless, the ratio of the shift $7.5 \times 10^{-4} / \mathrm{kbar}$ is smaller than that shown in Fig. 3, $1.0 \times 10^{-3} / \mathrm{kbar}$. Further, the half-width $3.5^{\circ}$ of the peak in $a$-Si does not change appreciably upon pressurizing (Ref. 5) contrary to the broadening shown in Fig. 2.

${ }^{30}$ K. Tanaka, Solid State Commun. 60, 295 (1986).

${ }^{31}$ B. Welber and M. H. Brodsky, Phys. Rev. B 16, 3660 (1977).

${ }^{32}$ F. Yonezawa and M. H. Cohen, in Fundamental Physics of Amorphous Semiconductors, edited by F. Yonezawa (Springer, Berlin, 1981), p. 119.

${ }^{33}$ D. Moses, A. Feldblum, E. Ehrenfreund, and A. J. Heeger, Phys. Rev. B 26, 3361 (1982); Y. Watanabe, S. Shichijyo, and K. Matsushige, Rep. Prog. Polym. Phys. Jpn. 30, 565 (1987). 